\title{
TAGUNG
}

\section{Der Balkan: eine Region im Wandel}

\author{
Frédéric Krumbein*
}

Die Konferenz beschäftigte sich mit den EUBeitrittsperspektiven der Balkanstaaten und deren innerer Entwicklung in ausgewählten Gebieten. Die Mitgliedschaft in der Europäischen Union ist nach Aussage von Olaf Leiße das erklärte Ziel aller Balkanstaaten.

\section{Zivilgesellschaftliche Entwicklungen und Partikularismus auf dem Balkan}

Anton Sterbling referierte in seinem Einführungsvortrag über die Zivilgesellschaft auf dem Balkan. Während der kommunistischen Herrschaft seien die vorhandenen zivilgesellschaftlichen Strukturen weitgehend zerstört worden. Nach dem Ende des Kommunismus stellte sich die Wiederherstellung zivilgesellschaftlicher Strukturen als schwierig dar. Ein verbreiteter Partikularismus in den Gesellschaften des Balkans behindere diese Etablierung und den Prozess der Modernisierung.

Die Geltung universell anerkannter Normen sowie Pluralismus sind nach Talcott Parsons ein Kennzeichen der Moderne. Partikularismus meine im Gegensatz dazu eine Abgrenzung gegenüber dem Anderen mit der Geltung eigener Normen und befördere Konformitätsdruck gegenüber den Angehörigen der eigenen Gruppe. Die Moderne zerstöre partikulare traditionelle Werte. Der Balkan befinde sich in einem Zustand partieller Modernisierung. Eine Erklärung für diesen Zustand partieller Modernisierung sei eine verspätete Nationalstaatsentwicklung nach dem Ende des Osmanischen und des Habsburger Reiches und eine in weiten Teilen noch agrarisch strukturierte Gesellschaft. Etatismus (eine starke und große Bürokratie), persönli-

\section{Die europapolitischen Perspektiven des Balkans}

Gemeinsame Tagung des Arbeitskreises Europäische Integration e.V., des Graduiertenkollegs 1412 „Kulturelle Orientierungen und gesellschaftliche Ordnungsstrukturen in Südosteuropa" und der Europa-Union Deutschland, Landesverband Thüringen

Jena, 19.-20. März 2010

Begrüßung

PD Dr. Olaf LEIßE, Friedrich-Schiller-Universität Jena

Prof. Dr. Klaus DICKE, Rektor der Friedrich-Schiller-Universität Jena

Einführungsvortrag: Zivilgesellschaftliche Entwicklungen und Partikularismus auf dem Balkan

Prof. Dr. Anton STERBLING, Hochschule der Sächsischen Polizei (FH), Rothenburg in der Oberlausitz

Panel I: Konsolidierung von Demokratie und Rechtsstaatlichkeit

Blockaden auf dem Balkan - kommt die EU an ihre Grenze(n)?

PD Dr. Bernhard STAHL, Heinrich-Heine-Universität Düsseldorf

Die Republik Moldau - Zwischen Westlichem Balkan und Östlicher Partnerschaft?

Dr. Anneli Ute GABANYI, Berlin

Panel II: Kultureller und sozialer Wandel

Regierungsautorität und Rechtsstaatlichkeit in Ländern des Westlichen Balkans

Dr. Martin BRUSIS, Ludwig-Maximilians-Universität München

Sprachnationalismus als Europäisierung?

Prof. Dr. Christian VOß, Humboldt-Universität zu Berlin

Abendvortrag: Die Republik Kosovo auf dem Weg nach Europa

S. E. Vilson MIRDITA, Botschafter der Republik Kosovo in Deutschland, Berlin

\footnotetext{
* Frédéric Krumbein, Geschäftsführer des Arbeitskreises Europäische Integration e.V., Berlin.
} 
che Beziehungen und Partikularismus führten dazu, dass in den Gesellschaften des Balkans kein Vertrauen in öffentliche Institutionen bestehe. Die Balkanstaaten seien Gesellschaften des öffentlichen Misstrauens, in denen Vertrauen nur in privaten Beziehungen existiere. Gesellschaftliche Solidarität setze aber ein weitverbreitetes Vertrauen in Staat und Gesellschaft voraus. Weitverbreitete Willkür der Verwaltung und die Besetzung von Verwaltungspositionen durch persönliche Beziehungen kennzeichneten hingegen den Balkan. Die staatlichen Ressourcen würden durch Machteliten ausgebeutet. Ziel der Eliten stelle die dauerhafte Sicherung ihres Machtmonopols dar. Während der kommunistischen Herrschaft habe es eine Verschränkung universalistischer und partikularer Strukturen gegeben. Die Werte besaßen einen universalistischen Anspruch, aber die Verteilung der Macht sei partikularistisch gewesen.

Der Partikularismus und seine Merkmale seien zentral für das Verständnis der heutigen Gesellschaften des Balkans. Selbst wenn diese Gesellschaften als Gesellschaften des öffentlichen Misstrauens bezeichnet werden könnten, so verfügten sie dennoch über aktive zivilgesellschaftliche Akteure. Die zukünftige Entwicklung hänge wesentlich davon $\mathrm{ab}, \mathrm{ob}$ sich universell anerkannte Normen in den $\mathrm{Ge}$ sellschaften des Balkans verbreiten. Kulturelle Normen veränderten sich nur langsam. Allerdings gebe es dynamisierende Elemente, wie die kapitalistische Wirtschaftsordnung und die Migration großer Teile der Bevölkerung, welche den Wandel von sozialen Strukturen und Werten beschleunigen.

Konsolidierung und Transformation politischer Systeme

Bernhard Stahl sprach über Hindernisse und Blockaden der Südosterweiterung der Europäischen Union. So blockierten die Niederlande und Belgien das Inkrafttreten des Stabilisierungs- und Assoziierungsabkommens mit Serbien. Der Grund liege in der mangelnden Kooperation mit dem Internationalen Strafgerichtshof für das ehemalige Jugoslawien. Ser-

\author{
Panel III: Mythen und die Konstruktion nationa- \\ ler Identitäten \\ Studierende und studentische Proteste im Serbien \\ der Ära Milošević \\ Đjorđje TOMIĆ, M.A., Berlin \\ Muslimische Erinnerungsorte und Geschichtsmy- \\ then in Bosnien-Herzegowina \\ Dennis DIERKS, M.A., Institut für Europäische \\ Geschichte, Mainz \\ Professional identities within a resistance culture: \\ teachers in Kosovo \\ Dr. Hazel-Ann SLINN, Friedrich-Schiller-Universi- \\ tät Jena \\ Podiumsdiskussion: Der Balkan - reif für die \\ EU? \\ Moderator: PD Dr. Olaf LEIßE, Friedrich-Schiller- \\ Universität Jena
}

bien erkenne außerdem das Kosovo nicht an, ebenso wie fünf EU-Staaten. Mazedonien streite sich mit Griechenland um den Namen des Staates.

Die Blockaden seien Ausdruck eines Identitätskonfliktes. Die Europäische Union sehe den Beitritt der Balkanstaaten als Erweiterung ihres eigenen zivilisatorischen Werteprojektes. Die Balkanstaaten betrachteten ihren Beitritt als kulturellen Anspruch zu Europa, zum europäischen Wertesystem, dazuzugehören. Der Balkan sei bis 1947 ein Spielball der Großmächte gewesen. Von 1948 bis 1991 sei Jugoslawien politisch weitgehend ignoriert worden. Die inneren Prozesse der Nationalisierung der einzelnen Ethnien ab den 1980er Jahren seien nicht wahrgenommen worden. Von 1991 bis 1999 sei Europa unfreiwillig in die Konflikte im ehemaligen Jugoslawien involviert worden. Seit 1999 engagiere sich Europa intensiv auf dem Balkan mit dem Ziel einer dauerhaften Stabilisierung. Seit dem EUGipfel in Thessaloniki im Jahr 2003 gäbe es eine klare Beitrittsperspektive für die Staaten des Westlichen Balkans.

Die Europäische Union selbst würde gerne ihre eigene Identität auf Südosteuropa übertragen. Die Identität Europas bestehe in der Abgrenzung zur eigenen Vergangenheit (Kolonialismus, Holocaust, Faschismus) und nicht in der Abgrenzung gegenüber anderen 
Großmächten. Dies sei einzigartig auf der Welt. Die Europäische Union glaube stark an die Kraft von Institutionen, an Solidarität und an Versöhnung als Basis ihrer Gemeinschaft.

Auf Seiten der Europäischen Union bereite neben der Erfüllung der Beitrittsbedingungen der Kandidatenländer das Kriterium der eigenen „Integrationsfähigkeit“ zunehmend Probleme für eine künftige Erweiterung. Der früher bestehende Konsens der immerwährenden Erweiterung sei einer „enlargement fatigue“ gewichen. Die Europäische Union habe die Osterweiterung noch als eine moralische Verpflichtung der Überwindung der historischen Teilung Europas angesehen. Die Erfahrung des ungeklärten Territorialkonfliktes im Fall Zypern sowie die großzügige Auslegung der Beitrittskriterien während des Beitritts von Bulgarien und Rumänien belasteten künftige Beitritte.

Auf der Seite der Balkanstaaten sei zu konstatieren, dass diese schwache Staaten mit schwachen Institutionen darstellten. Die politischen Systeme zeichneten sich durch „Demokratien ohne Demokraten“ aus. Die Nationalstaaten seien verspätet gegründet worden und hätten ein unreflektiertes Verhältnis zur eigenen Vergangenheit. Nationalisierte Geschichtspolitik und eine nationalisierte Erinnerungskultur prägten das Verhältnis zur eigenen Vergangenheit.

Anneli Ute Gabanyi sprach über die Republik Moldau und die vielfältigen Probleme dieses Staates. Die Republik Moldau verfüge über Grenzprobleme mit Rumänien und der Ukraine und sei wirtschaftlich stark abhängig von Russland. Russland sehe Moldau als seine Einflussregion. Die nationale Identität bleibe unklar: rumänisch oder ,moldauisch"? Das größte Sicherheitsproblem stelle das abgespaltene Transnistrien dar: Moldau übe keine vollständige Kontrolle über die eigenen Grenzen und das eigene Territorium aus.

\section{Politischer und kultureller Wandel}

Martin Brusis stellte die demokratische und rechtsstaatliche Situation in den Ländern des
Westlichen Balkans vor. In allen Ländern sei die Demokratie mehr oder weniger stark konsolidiert. Freie und faire Wahlen fänden regelmäßig statt, es gäbe Regierungswechsel und die Demokratie werde von keinem Akteur offen infrage gestellt. Die Zustimmung zur Demokratie sei in den Balkanstaaten relativ hoch (56,4 Prozent im Jahr 2006).

Defizite bestünden im Bereich der Pressefreiheit. Hier gäbe es teilweise gravierende Einschränkungen, wie die Ermordung von Journalisten in Kroatien. Der Medienmarkt sei oftmals konzentriert und in der Hand weniger politisch und/oder gesellschaftlich einflussreicher Akteure. Die Auswahl der Richter zeichne sich teilweise durch eine starke Politisierung aus. Korruption sei weitverbreitet. Die Finanzierung der Parteien werde oft nicht unabhängig kontrolliert. Die oft gravierenden Defizite im Bereich des Rechtsstaats hätten vielfältige Ursachen: Lückenhafte Gesetze, fehlende beziehungsweise unzureichend qualifizierte Beamte oder Richter, mangelnde finanzielle Ressourcen, eine schwache Zivilgesellschaft, die wirtschaftliche Abhängigkeit von Journalisten, schwache öffentliche Kontrollen der Medien und konfrontative Politikmuster in der politischen Kultur. Eine wichtige, aber oft vernachlässigte Ursache sei der Schutz von Patronagenetzwerken. Regierende Parteien nutzten staatliche Ressourcen zur Belohnung ihrer Anhängerschaft (,state capture'). Ein robuster Parteienwettbewerb könne als einziges Mittel eine solche Ausbeutung des Staates verhindern.

Im Parteiensystem der Balkanstaaten seien positiv eine organisatorische Kontinuität gröBerer Parteien und eine geringere Fragmentierung vieler Parteiensysteme zu beobachten. Negativ seien polarisierte Parteienwettbewerbe (Albanien) und eine dominante Regierungspartei (Montenegro). Der Parteienwettbewerb beschränke sich teilweise auf ethnische Zugehörigkeit (Mazedonien, Bosnien). Die besten Chancen für einen robusten Parteienwettbewerb bestünden in Kroatien und Serbien. Die wachsende Regierungsauto- 
rität ohne einen robusten Parteienwettbewerb begünstige die Einmischung der Exekutive in die Legislative, die Verwaltung, die Jurisdiktion sowie die Medien. Der EU-Beitrittsprozess stärke durch einen Informationsvorsprung ebenfalls die Exekutiven.

Christian Voß referierte über die Sprachen des Balkans und ihre Rolle im Prozess der Europäisierung. Das Habsburger Reich und Russland konkurrierten um den Balkan, um die südslawischen Sprachen und deren Zugehörigkeit zur europäischen oder zur russischen Sprachsphäre.

Sprachnationalismus als Modell einer homogenen Sprachverwendung in einem Land habe auf dem Balkan katastrophale Auswirkungen gehabt. In Slowenien bemühe man sich um eine Reinhaltung der Sprache, wie eine Ersetzung der Anglizismen durch slowenische Wörter. Die Kroaten bezeichneten ihre Sprache als kroatisch, nicht als serbokroatisch. Die Bulgaren wollten sich sowohl gegenüber den Türken als auch gegenüber den Griechen (mit denen sie die gleiche Religion teilen) abgrenzen und haben ihre Schriftsprache latinisiert.

Sprache und ihre Verwendung drückten die eigene individuelle Identität aus. Bewohner des Balkans, die sich emotional stark an Europa gebunden fühlen, verwenden teilweise andere Wörter als Bewohner, die sich vor allem als Balkanbewohner sehen.

Sozialer Wandel und die Konstruktion nationaler Identitäten

Đjorđje Tomić referierte über studentische Proteste in Serbien während der Ära Milošević. Slobodan Milošević kam Ende der 1980er Jahre an die Regierung. Serbien unter Milošević sei semiautoritär, nationalistisch und international isoliert gewesen. Proteste von Studierenden gegen das Regime gab es 1991, 1992 und 1996/1997 in allen serbischen Universitätsstädten. 1992 forderten die Studierenden beispielsweise die Aufhebung der Kontrolle der Medien, den Rücktritt von
Milošević und Autonomie für die Universitäten.

Die Proteste hätten mehrere Gemeinsamkeiten gehabt: Die Organisationsstrukturen und Kommunikationsstrategien gegenüber der Öffentlichkeit seien sehr gut gewesen. Studierende hätten mehr Demokratie gefordert. Die Studierenden hätten Demokratie oberflächlich definiert. Freie und faire Wahlen und bestenfalls noch Medienfreiheit konstituierten den Kern des studentischen Demokratiebegriffs. Die Proteste hätten an den politischen Kontext und den dominanten nationalistischen Diskurs angeknüpft, auch wenn sie nicht eindeutig nationalistisch gewesen seien. Die Proteste entwickelten im Laufe der 1990er Jahre zunehmend ein Ziel: den Sturz von Milošević. Grundlegende soziale Fragen seien von den Studierenden größtenteils ignoriert worden.

Dennis Dierks sprach über muslimische Erinnerungsorte und Geschichtsmythen in Bosnien-Herzegowina. Die Erinnerung als autonomes Reich könne dem Menschen nicht genommen werden und könne als Zufluchtsort aus einer unliebsamen Gegenwart dienen. „Ein Volk, das seine Ahnen ehrt, träumt in der Vergangenheit seine Zukunft", so die Formulierung eines kroatischen Dichters. Die Zeit hinterlasse in der Geschichte jedes Volkes Wunden, aber die Erinnerung an Volkshelden lindere diese Wunden.

Die bosnischen Muslime hätten sich gegenüber zwei Imperien verorten müssen: Dem Osmanischen Reich und Österreich-Ungarn. Im Osmanischen Reich hätten die muslimischen Bosnier eine wichtige Rolle gespielt. Sie hätten sich deshalb gegen eine Annexion durch Österreich-Ungarn gewandt. Die Kroaten und die Serben, die in Bosnien wohnten, hätten versucht die Muslime auf ihre Seite zu ziehen, da keine der beiden Gruppen eine Mehrheit bildete. Die Muslime hätten sich diesen Versuchen verweigert. Während der österreichisch-ungarischen Herrschaft hätten die Habsburger versucht eine eigene $\mathrm{Ge}$ - 
schichte und Identität Bosnien-Herzegowinas zu konstruieren, um zu verhindern, dass die Bosnier sich am kroatischen oder serbischen Nationalismus beziehungsweise am Osmanischen Reich orientieren. Dieser Versuch sei gescheitert. Jugoslawien hätte die bosnischen Muslime als drittes Staatsvolk Bosnien-Herzegowinas neben Kroaten und Serben anerkannt. Aktuell bestehe kein allgemein akzeptiertes Narrativ zur Verortung der bosnischen Muslime in der Gegenwart. Ein solches Narrativ müsste dem multikulturellen Charakter Bosniens sowie der islamischen Geschichte des Landes gerecht werden. Eventuell könnte das Konzept des „Euroislam“ diese Funktion erfüllen.

Hazel-Ann Slinn stellte Erfahrungen von Lehrerinnen und Lehrern im Kosovo dar. Sie hat narrative Interviews mit Lehrerinnen und Lehrern geführt und nach deren Erfahrungen während der serbischen Herrschaft und während des Kosovokrieges gefragt. Seit den 1980er Jahren habe es im Kosovo ein paralleles Bildungs- und Gesundheitssystem (ein offizielles serbisches und ein inoffizielles albanisches) gegeben, weil unter den Albanern kein Vertrauen in die staatlichen Systeme herrschte. Es habe eine weitverbreitete Diskriminierung im Bildungssystem stattgefunden. Kosovoalbaner hätten keinen oder nur eingeschränkten Zugang zum Bildungssystem gehabt. Die Kosovoalbaner hätten teilweise auf Formularen deklarieren müssen, dass sie keine Albaner waren, um Zugang zu Schulbildung zu bekommen. Die Curricula seien ein- seitig auf die serbische Mehrheit Serbiens ausgerichtet gewesen. Die albanischen Lehrer im parallelen Bildungssystem hätten kaum Geld erhalten (nur aus der albanischen Diaspora und von Nachbarn aus den Ortschaften).

\section{Ist der Balkan reif für die EU?}

Ist die Mitgliedschaft in der Europäischen Union überhaupt positiv für die Balkanstaaten, weil die Erfahrungen zeigen, dass der Reformwille nach einem Beitritt meist erlahmt? Dies fragte Olaf Leiße in der abschließenden Diskussion. Bernhard Stahl antwortete, aus ökonomischer Perspektive sei für alle Balkanstaaten deutlich, dass es für ihre Volkswirtschaften keine Alternative zur Europäischen Union gäbe.

Christian Voß ergänzte bezüglich einer Erweiterung der Europäischen Union, dass eine gemeinsame Wertebasis notwendig sei. Am Beispiel Griechenlands in der Finanzkrise zeige sich die mangelnde Solidarität innerhalb der Europäischen Union. Die Vertiefung der Europäischen Union sei in den letzten Jahren zu stark vernachlässigt worden.

Hazel-Ann Slinn fügte hinzu, dass die Kultur des Balkans eine eigene sehr wertvolle Kultur darstelle, die sich nicht an die nordeuropäische Kultur anpassen sollte. Der Balkan besitze positive Werte wie Gastfreundschaft und Zeit für Freunde und Familie, die in den nordeuropäischen Gesellschaften so nicht mehr existierten. 